\title{
Structural prediction of graphitization and porosity in carbide-derived carbons
}

\author{
Carla de Tomas \\ Department of Physics and Astronomy, Curtin University, Perth WA 6102, Australia \\ Irene Suarez-Martinez \\ Department of Physics and Astronomy, Curtin University, Perth WA 6102, Australia \\ Fernando Vallejos-Burgos \\ Center for Energy and Environmental Science, Shinshu University, Wakasato, Nagano 380-8553, Japan \\ Maria J. Lopez \\ Department of Theoretical Physics, Atomic Physics and Optics, Universidad de Valladolid, 47005 Valladolid, Spain \\ Katsumi Kaneko \\ Center for Energy and Environmental Science, Shinshu University, Wakasato, Nagano 380-8553, Japan \\ Nigel A. Marks* \\ Department of Physics and Astronomy, Curtin University, Perth WA 6102, Australia
}

\begin{abstract}
Carbide-derived carbons (CDCs) are nanoporous carbons with a tunable pore size, making them desirable for their adsorption properties. Despite their applicability, reliable structural models are difficult to construct due to the interplay between strong short-range order and long-range disorder. Here, a mimetic methodology is developed to generate atomistic models of CDCs using Molecular Dynamics and the Environment Dependent Interaction Potential. This approach reproduces the main characteristics of experimentally-prepared CDCs, including microstructure, porosity at the nanometre scale, and graphitization with increasing temperature. An Arrhenius-based approach is used to bridge the timescale gap between Molecular Dynamics and experiment and build a connection between the simulation and synthesis temperatures. The method is robust, easy to implement, and enables a fast exploration of the adsorption properties of CDCs.
\end{abstract}

\section{Introduction}

Carbide-derived carbons (CDCs) are an important class of nanoporous carbons with a wide range of potential applications including gas separation, hydrogen storage, catalyst supports and electrodes

*Corresponding author. Tel: 0061 89266-1386. E-mail: N.Marks@curtin.edu.au (Nigel Marks)

Preprint submitted to Elsevier in batteries. $[1,2,3]$ The advantage of CDCs over other porous materials is that the pore size distribution (PSD) is narrow and tunable through the choice of precursor material and processing conditions. [4] A wide range of binary and ternary carbides can be used as precursor, including $\mathrm{ZrC}, \mathrm{TiC}$, $\mathrm{B}_{4} \mathrm{C}, \mathrm{SiC}, \mathrm{Ti}_{2} \mathrm{AlC}$ and $\mathrm{Ti}_{2} \mathrm{SiC}$. CDCs are produced by extracting the non-carbon species, most typically by halogenation using chlorine, accompanied

March 22, 2017 
by thermal annealing to $400-1200{ }^{\circ} \mathrm{C}$. [2] The effect of temperature on the microstructure has been extensively studied using transmission electron microscopy (TEM), Raman spectroscopy and X-ray diffraction (XRD). [5, 6, 7, 8] At low temperatures, all carbides transform into a disordered microstructure, and with increasing temperature different levels of graphitization are observed. [2] Many carbide precursors, such as $\mathrm{ZrC}, \mathrm{Fe}_{3} \mathrm{C}$ and $\mathrm{TiC}$, produce graphite-like ribbons upon heat treatment above $1000{ }^{\circ} \mathrm{C},[5,6,7,9]$ while other precursors, such as $\mathrm{SiC}$ will only graphitize in the presence of a metallic catalyst. [10]

Atomistic models of CDCs are valuable from both a conceptual and a practical point of view. They are highly sought after as they provide a visual representation of the structure and enable direct simulation of gas adsorption and other properties. [11, 12] These models, which overlap with nanoporous carbons, can be divided into two groups: geometric models and simulation models. Geometric models are highly idealized and are often constructed by hand. The simplest geometric model is the slit-pore model, [13] comprising two infinite graphene sheets. While widely used, $[13,14,15,16,17,18]$ it neglects fundamental features such as curvature, edges and pore-size distribution. Improvements to the slit-pore model introduce defects [19] and/or three-dimensional networks. [20, 21] More complex geometric methods employ fullerenic [22] or polymeric [23] fragments to create a three-dimensional structure.

Simulation models are in principle more realistic than geometric models and generate the atomic positions by either mimicking the experimental synthesis or reconstructing the coordinates using experimental data. [24] Reconstructive methods based on Reverse Monte Carlo (RMC) [25, 26, 27, 28, 29, $30,31]$ use scattering data as input and solve an inverse problem in which the atomic coordinates are evolved until they reproduce this data. While they are not predictive, RMC methods have the advantage that the models can reproduce experimental properties associated with morphology and topology. Typically the RMC algorithm is augmented by an interatomic potential to avoid unphysical structures, in particular an excess of triangles and quadrilaterals. This approach is known as Hybrid RMC (HRMC) [25] and has been successful in the modelling of CDCs [31] and other nanoporous carbons. [26, 27, 28] Drawbacks of these reconstructive methods include the need for high-quality ex- perimental data to facilitate the inversion, the inability to predict structures for conditions where experimental data does not exist, and the lack of structural uniqueness associated with the inversion problem itself.

The predictive capability of mimetic methods is an attractive alternative for the simulation of CDCs. Experimental data is not required as input and so simulations can be used to foresee new conditions. As noted in the recent review by Bhatia [24], the biggest challenges for mimetic methods are the timescale mismatch (nanoseconds in simulations versus hours in experiments) and the suitability of the carbon potential. There have been a number of mimetic approaches using $\mathrm{MD}$ and Monte Carlo (MC) to generate nanoporous structures. The most well-known model is from Palmer et al. [32] who performed an extensive study of CDC synthesis using Quench Molecular Dynamics. Their starting structure is a carbon liquid which is quenched at various rates to form a solid. With slower quench rates, the structures become more ordered and develop a porous structure. However, they do not observed the stacking of layers associated with graphitization. They suggest [32] this is due to their use of a reaction-state-summation (RSS) potential [33] which lacks long-range dispersive attraction between layers. Additionally, the RSS potential neglects $\mathrm{sp}^{3}$ bonding, preventing three-dimensional junctions like merging of sheets. Peng and Morris [34] employed a similar approach to Palmer et al. and used a more accurate tightbinding potential but the quenching rate was 50 times faster, resulting in a largely amorphous structure. Another related approach uses metropolis $\mathrm{MC}$ in combination with quenching of a liquid to generate coordinates. $[35,36]$ These structures are broadly similar to those produced by Palmer et al. with slow quench rates, and none exhibit graphitization.

An alternative mimetic strategy involves annealing instead of quenching. The advantage of this approach is that the thermal treatment step in the experimental synthesis of CDCs is modelled in the simulation by an explicit temperature, rather than a quenching rate. An annealing-based mimetic MD approach was first used by Lopez et al. [37] using the Tersoff-Nordlund potential [38] which permits both $\mathrm{sp}^{2}$ and $\mathrm{sp}^{3}$ bonding and has long-range attraction. They used long annealing times (up to $2 \mathrm{~ns}$ ) and large system sizes (up to 62,500 atoms), and reproduced qualitative trends associated with 
the skeleton of the structure and the porosity. However, graphitic stacking as seen in experiments was not observed and there was no link between the experiments and the high MD temperatures used for the annealing. Recently, Ranganathan et al. [39] used a two-stage MD methodology commencing with quenching followed by high temperature annealing. Even though the potential they used (ReaxFF [40]) includes a long-range attractive term, stacking of layers was not observed.

In this work we present an annealing-based mimetic MD approach and apply it to generate realistic TiC-CDC structures. Our simulations use the Environment Dependent Interatomic Potential (EDIP) [41] for carbon. EDIP has been successful in MD simulations of graphitization processes $[42,43,44,45,46]$ and in HRMC and MC models of nanoporous carbon. [36, 30, 47, 31] To bridge the gap between the experimental and simulation timescale we develop an Arrhenius framework which allows correlation between the experimental and simulation temperatures. In agreement with experiments, we observe that at low temperature the structures are disordered and as temperature increases graphitic fragments develop, forming stacks at the highest temperatures. We compare our results with a variety of experimental data (TEM images, pair distribution functions, electron energy loss spectroscopy (EELS) and absorption isotherms) and find a substantial improvement over previous mimetic models.

\section{Methodology}

\subsection{Simulation methods}

The Molecular Dynamics simulations are performed using the Environment Dependent Interatomic Potential for carbon (EDIP) [41] embedded in the LAMMPS software. [48] Recent work has shown EDIP is the most suitable carbon potential to model the annealing of low density amorphous carbons. [42] Graphitization has also been successfully modeled in $0 \mathrm{D}, 1 \mathrm{D}, 2 \mathrm{D}$, and 3D carbon structures using the EDIP potential. [43, 45, 46]

We employ an annealing methodology in which the simulation temperature is maintained at a single value for a long period. This method is different from Quench Molecular Dynamics where a liquid structure is rapidly cooled down at different rates. Our methodology mimics the experimental synthesis of TiC-CDC. Starting from a TiC precursor, Ti atoms are removed, leaving behind a carbon facecentered cubic sublattice with a lattice constant of $0.433 \mathrm{~nm}$ and a density of $0.98 \mathrm{~g} / \mathrm{cc}$. The sublattice is annealed for $2 \mathrm{~ns}$ at temperatures of 1500,2000 , $3000,4000 \mathrm{~K}$ and for $10 \mathrm{~ns}$ at $4500 \mathrm{~K}$. Temperature control is achieved using the Bussi thermostat [49] and all calculations are performed using a timestep of $0.2 \mathrm{fs}$ and periodic boundary conditions. Density is kept constant using a fixed cubic box of $10.4 \mathrm{~nm}$ length and 55,296 atoms. The annealed structures contain a small number of triangles, most of which are detached from the structure, involving approximately $0.015 \%$ of the atoms at 2000 and $3000 \mathrm{~K}$ and $0.3 \%$ at 1500 and $4000 \mathrm{~K}$. These atoms are removed from the system at the end of the annealing cycle. Energy minimization using a conjugate gradient scheme is performed prior to all structural analysis. This includes removal of triangles, snapshots, coordination numbers, ring statistics, radial distribution functions and adsorption isotherms. Coordination numbers are measured by counting neighbours within a cutoff of $1.85 \AA$. Shortest-path ring statistics are calculated using the algorithm of Franzblau [50], using an inhouse code previously used to study amorphous carbon [51]. Images are rendered using the OVITO software [52].

Isotherms are calculated using Grand Canonical Monte Carlo (GCMC) simulations at $77 \mathrm{~K}$. Each simulation is run in periodic boundary conditions using the coordinates of the annealed CDC structures. The MC moves are creation, annihilation and displacement of $\mathrm{Ar}$ atoms with equal probability. Atomic interactions are modeled using an unshifted Lennard-Jones $12-6$ potential with parameters $\sigma=0.3405 \mathrm{~nm}$ and $\epsilon=120.0 \mathrm{~K}$ for $\mathrm{Ar}-\mathrm{Ar}$ and $\sigma=0.338 \mathrm{~nm}$ and $\epsilon=58.0 \mathrm{~K}$ for the Ar-C cross interactions. [53] The cut-off distance is set at $1.5 \mathrm{~nm}$. Each adsorption pressure point is sampled for 10 million steps and the first 5 million steps are discarded to ensure equilibration. Poresize distribution (PSD) functions are calculated using two methods: (i) a geometric method, and (ii) Non-Local Density Functional Theory (NLDFT) using the GCMC isotherms. For the geometric method, pores are measured as non-overlapping spheres placed in the structure voids with an exclusion radius of $1.7 \AA$. [54, 37] Pore size is determined by the sphere diameter. The NLDFT calculations are performed using the ASiQwin software from Quantachrome [55]. 


\subsection{Comparison with experiment}

In this work we compare our simulations to TiCCDC experiments from multiple authors. The main comparison is with TEM, isotherm and PSD data from Gogotsi's group as reported in Refs. [6], [7] and [9]. They performed a three hour chlorination at $600,800,1000$ and $1200^{\circ} \mathrm{C}$. With increasing temperature they observed a gradual evolution from a disordered arrangement towards a more graphitic microstructure. Recently, similar experiments raising the chlorination temperature above $1200{ }^{\circ} \mathrm{C}$ have been reported by several groups. $[8,10,56]$ The most comprehensive high-temperature study is by Gläsel et al. [8] who annealed up to $1585{ }^{\circ} \mathrm{C}$, observing substantial graphitization and $8 \mathrm{~nm}$ mesopores. EELS data from Gläsel et al. is compared against the simulation coordination fractions. Pair distribution functions (PDFs) are compared to the recent work by Forse et al. employing X-ray diffraction. [57] Their experimental data is processed by Fourier transforming with a value of $Q_{\max }=24 \AA$, leading to significant broadening of peaks in the PDF. When comparing with experimental data, the PDF from the simulations was convolved with a Gaussian of width $0.11 \AA$. This value reproduces the height and width of the first neighbour peak of a graphite nanocrystal as presented in Fig. S3 of the supplementary material in Ref. [57].

\subsection{Mapping between simulation and experimental temperatures}

A major challenge in linking MD simulations with experiments is the large disparity in timescale between the two. [24] MD simulations are typically on the nanosecond scale, around 13 orders of magnitude shorter than the experimental annealing time. In this work we overcome this mismatch using the simple technique of temperature acceleration. [58] As we will demonstrate, this approach provides a good representation of the temperature effect seen in the experimental data. The first step is to assume Arrhenius behavior

$$
f=A \exp \left[-E_{a} / k_{B} T\right]
$$

with a single activation barrier $E_{a} ; f$ is the frequency of events, $A$ is the attempt frequency, $T$ is the temperature and $k_{B}$ is Boltzmann's constant. The correspondence between the experimental and simulation temperatures is determined by equating the time-frequency product (i.e. $f_{\text {expt }} \times t_{\text {expt }}=$ $\left.f_{\operatorname{sim}} \times t_{\mathrm{sim}}\right)$ to ensure that the same number of events occur in both simulation and experiment. This yields the expression

$$
T_{\text {sim }}=-\frac{E_{a}}{k_{B}} \times\left[\log \left(\frac{t_{\text {expt }}}{t_{\text {sim }}}\right)-\frac{E_{a}}{k_{B} T_{\text {expt }}}\right]^{-1}
$$

which relates the experimental temperature and time $\left(T_{\text {expt }}\right.$ and $\left.t_{\text {expt }}\right)$ to the simulation temperature and time $\left(T_{\operatorname{sim}}\right.$ and $\left.t_{\text {sim }}\right)$. It is worth noting that the Arrhenius expression in Eq. 2 involves the logarithm of the ratio of the times, and therefore a substantial variation in time produces only a small change in the temperature. For example, if the experimental annealing time were $6 \mathrm{hr}$ instead of $3 \mathrm{hr}$, the changes in equivalent simulation temperature would be at most $150 \mathrm{~K}$.

Figure 1 plots Equation 2 for barriers of 5.5, 6.0 and $6.5 \mathrm{eV}$ for the case of an experimental time of $10^{4} \mathrm{~s}$ and a simulation time of $2 \mathrm{~ns}$. The value of $t_{\text {expt }}$ is chosen based on the experiments from Gogotsi's group, [7] while the activation barriers were selected post hoc on the basis of similarity between the TEM images and our simulated structures. The dotted vertical lines at 600, 800, 1000 and $1200{ }^{\circ} \mathrm{C}$ indicate the temperatures used in Gogotsi's experiments, and the shaded areas show the corresponding range of simulation temperatures. Full circles show the simulation temperatures used in this work; these are $T_{1}=1500 \mathrm{~K}$, $T_{2}=2000 \mathrm{~K}, T_{3}=3000 \mathrm{~K}$ and $T_{4}=4000 \mathrm{~K}$. To compare with an additional data point from Gläsel et al. [8], we also perform a simulation at $T_{5}=4500 \mathrm{~K}$ running for $10 \mathrm{~ns}$.

\section{Results and Discussion}

Figure 2 compares our simulations and TEM images of TiC-CDCs synthesized at five different temperatures; both sets of images have the same length scales. The first four experimental images (panels a-d) are from Gogotsi's group while the fifth (panel e) is from Glässel et al. The sequence of TEM images shows a gradual graphitization as the chlorination temperature increases and the simulated structures exhibit the exact same behavior. At the lowest temperature the experimental image and simulated structure are essentially fully disordered, with uniform and relatively small pores. At $800{ }^{\circ} \mathrm{C}$ order begins to emerge in the form of curved graphene sheets as can be seen by the contrast in the micrograph. The same curved structures are seen in the simulation at temperature $T_{2}$ and larger pores 


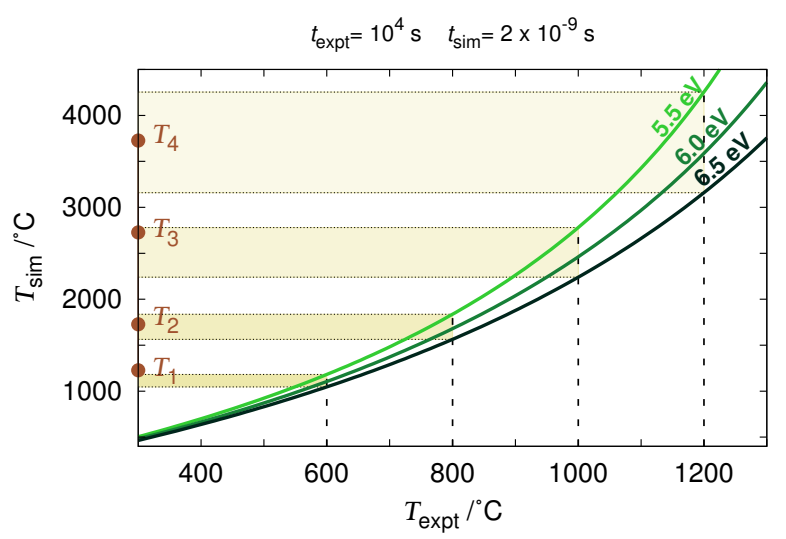

Figure 1: Correspondence between experiment and simulation temperatures according to Equation 2 using activation energies of 5.5, 6.0 and $6.5 \mathrm{eV}$ for experiments taking about $3 \mathrm{hr}$ and simulation times of 2 ns. Dotted vertical lines indicate the temperatures used in the experiments from Ref. 7]. For each experimental value, a shaded area shows the temperature range at which the simulation can be performed assuming the given activation energies. Full circles show the simulation temperatures used in this work, i.e. $T_{1}=1500 \mathrm{~K}$, $T_{2}=2000 \mathrm{~K}, T_{3}=3000 \mathrm{~K}$ and $T_{4}=4000 \mathrm{~K}$.

start to develop. At $1000{ }^{\circ} \mathrm{C}$ the graphene sheets are longer and straighter, with some stacking. Our simulation at $T_{3}$ also shows larger graphene sheets, but stacking is absent. At $1200{ }^{\circ} \mathrm{C}$ the experiments exhibit curved, thin graphitic ribbons and the corresponding simulations find similar stacked structures. At the highest temperatures, a large number of stacked layers, as many as 20, are observed experimentally. The simulated structures also contain a large number of layers, though not as many due to the finite size of the box.

The strong similarities seen in Figure 2 justifies the Arrhenius model presented above. For the comparisons involving Gogotsi's data, there is excellent correlation between the experimental synthesis temperature and the associated temperatures $T_{1}$, $T_{2}, T_{3}$ and $T_{4}$. There is no a priori reason why they should correlate so well, but the fact that they do indicates that annealing of CDCs can be well described by a single activation barrier. The magnitude of the activation barrier is similar to rearrangements occurring at the atomic scale such as Stone-Wales rotation, interstitials and vacancies, with formation energies of $\sim 5.2,5.8$ and $7.5 \mathrm{eV}$ respectively. [59] Note that these atomic activation barriers should not be compared to activation barriers calculated from experimental rate constants
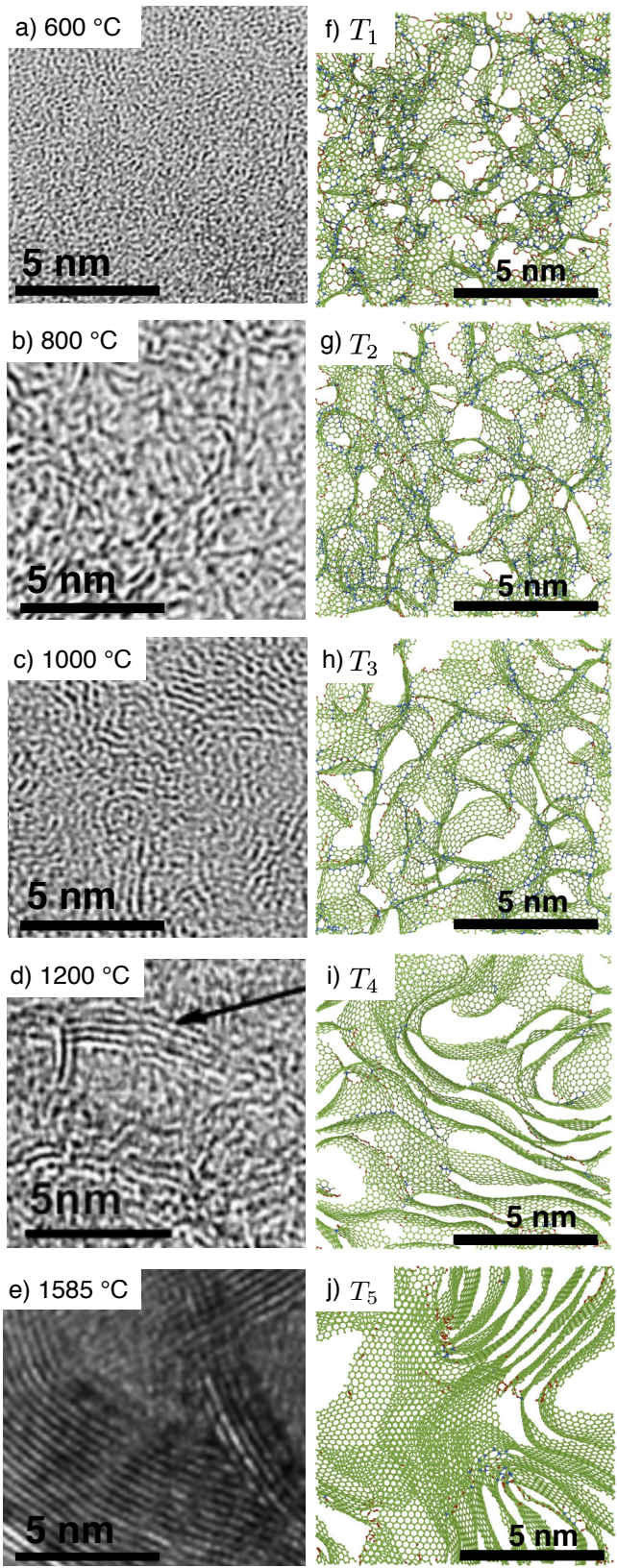

Figure 2: a-e) TEM images of TiC-CDCs obtained by chlorination at various temperatures. f-i) Slab of $2 \mathrm{~nm}$ thickness of simulated TiC-CDCs after annealing at $T_{1}=1500 \mathrm{~K}$, $T_{2}=2000 \mathrm{~K}, T_{3}=3000 \mathrm{~K}, T_{4}=4000 \mathrm{~K}$ and $T_{5}=4500 \mathrm{~K}$. Red, green and blue spheres denote $s p, s p^{2}$ and $s p^{3}$ bonding, respectively. Panel a) reprinted with permission [9]. Copyright 2006 Elsevier Ldt. Panels b-d) reprinted with permission [7]. Copyright 2006 John Wiley and Sons. Panel e) reprinted with permission [8]. Copyright 2015 American Chemical Society.

which are typically $0.4-1.0 \mathrm{eV}$. [2] While our approximation of a single atomic activation barrier is 
a considerable simplification of the process, this relationship provides a useful guide to correlate simulations and experiments.

The simulation performed at temperature $T_{5}$ is at the upper limit of what it can be achieved with temperature acceleration. At $4700 \mathrm{~K}$ the structure melts and phase-separates into a liquid and a gas and therefore calculations near and above this temperature cannot be performed. As an aside, this phase-separation and associated clustering occurs in the ReaxFF simulations by Ranganathan et al. [39] where the liquid carbon is heated to $10,000 \mathrm{~K}$. In our simulations, to mimic an experimental temperature of $1585{ }^{\circ} \mathrm{C}$ there are two possibilities: (i) a simulation time of 2 ns with a temperature of approximately $8000 \mathrm{~K}$, or (ii) a very long simulation of the order of microseconds at a temperature of $4500 \mathrm{~K}$. The former is beyond the melting point and hence impossible, while the latter is computationally too expensive. As a compromise, we anneal at $T_{5}=4500 \mathrm{~K}$ for $10 \mathrm{~ns}$. Despite this approximation, there is good qualitative agreement between experiment and simulation in Figures $2 \mathrm{e}$ and $2 \mathrm{j}$, with the simulations reproducing large stacks of graphene sheets which are not evident at $T_{4}$.

The effect of temperature on CDC synthesis has not previously been modeled with the accuracy seen in Figure 2. Although a large number of methodologies and carbon potentials have been applied, no previous work exhibits the stacking of graphitic layers or the correlation with experimental synthesis conditions. Palmer et al. [32] also present a sideby-side comparison with TEM images and observe some correlation with experimental data, but they do not observe any graphitization (see Fig. 3C in Ref. [32]). Additionally, their study uses the liquidquenching rate as a proxy for annealing, and hence there can be no mapping between the simulation parameters and the experimental conditions.

The strength of our approach is demonstrated in Figure 3 which presents coordination fractions and ring statistics as a function of simulation temperature. In panel (a) we compare EELS data from Gläsel et al. [8] with our simulations and see excellent agreement. EELS measures the $\pi^{*}$ peak which has contributions from $s p$ and $s p^{2}$ bonding, and hence the simulation quantity plotted is the sum of the two. At $T_{1}$ and $T_{2}$ the $s p$ contribution is 10.0 and $6.6 \%$ respectively, while at high temperatures the $s p$ contribution drops to $\sim 1 \%$. The dominant contribution is from $s p^{2}$ bonding, giving an average

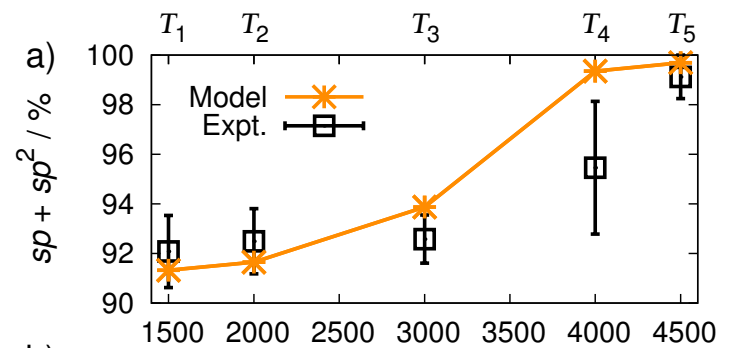

b)
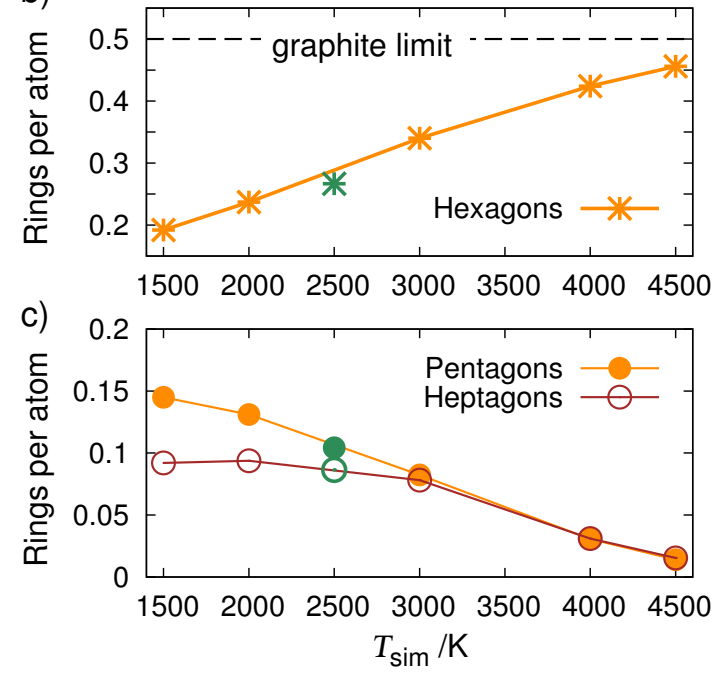

Figure 3: a) Variation of $s p+s p^{2}$ fraction with temperature provided by our simulations. Experimental data from Gläsel et al. [8] at $600,800,1000,1200$ and $1585^{\circ} \mathrm{C}$. b) Variation of number of hexagons per atom with simulation temperature. c) Number of pentagons and heptagons per atom as a function of simulation temperature. Green symbols in b) and c) are from a $1 \mathrm{~g} / \mathrm{cc}$ MC simulation by Furmaniak. [36]

coordination number between 2.95 and 3.05 across all temperatures, consistent with the graphitic nature of CDCs. In contrast, Palmer et al. report coordination numbers as low as 2.0, corresponding to mainly $s p$ bonding; this structure is unphysical and consists mainly of linear chains and small fragments. More reasonable coordination numbers between 2.5 and 2.9 are reported by Peng and Morris, [34] most probably due to their more accurate tight-binding potential.

Panels (b) and (c) in Figure 3 demonstrate that our structures become increasingly graphitic with temperature, with the number of hexagons almost reaching the graphite limit and the number of heptagons and pentagons tending to zero. At the lowest temperatures, $T_{1}$ and $T_{2}$, there is an excess of pentagons over heptagons, and the pore walls are small interconnected polyaromatic fragments. For 
all other temperatures, the number of pentagons and heptagons are equal, and hence there is no spherical curvature according to the Euler criterion. This can be seen in Figure 2, where the platelets become larger and more planar as temperature increases. Despite a hexagon count similar to this work, Lopez et al. (using a Tersoff-Nordlund potential) report an excess of heptagons over pentagons at all temperatures, resulting in a Schwarzite-like structure with negative curvature. These structures are incompatible with the planar graphitic stacks observed experimentally. A similar excess of heptagons over pentagons is seen in the ReaxFF simulations of Ranganathan et al. [39] Furmaniak [36] performed liquid quenching MC simulations with EDIP [41] (the same potential as this work) and as shown in Figure 3, the resulting structure has ring statistics that can be placed between $T_{2}$ and $T_{3}$, equivalent to an experimental temperature around $900{ }^{\circ} \mathrm{C}$. This similarity provides reassurance that the crucial factor is the potential itself: despite using two different methods, the MD and MC approaches predict similar final structures.
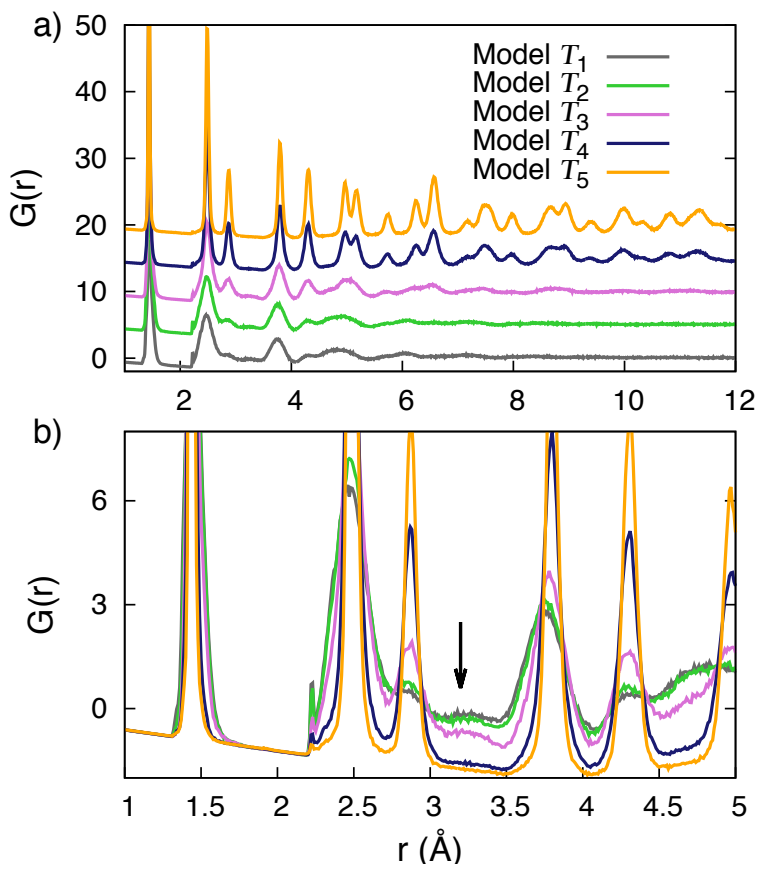

Figure 4: Pair distribution functions of the five simulated structures, showing increasing order as the annealing temperature increases. a) Raw data showing long-range ordering at high temperature; data is offset by 5 atoms $/ \AA^{2}$. b) The same data over a smaller range and with no offset. The arrow indicates distance associated with heptagons as described in text.
Figure 4 shows pair distribution functions (PDFs) for our five structures. In panel (a) the PDFs are offset by 5 atoms $/ \AA^{2}$ from each other for clarity, while in panel (b) we show a close-up view of the first six peaks. The PDF is defined as $G(r)=4 \pi r\left(\rho(r)-\rho_{0}\right)$, where $\rho(r)$ is the density of atoms at a distance $r$ from an arbitrary atom and $\rho_{0}$ is the average atomic density. As the temperature increases, the degree of long-range order increases markedly, extending beyond $15 \AA$ for model $T_{5}$. This increased order is associated with a pronounced sharpening of all peaks as the local structure begins to more closely resemble graphite. In recent experiments by Forse et al. [57], they noticed a small feature in $G(r)$ near $3.2 \AA$ which they attributed to heptagons. They suggested that the reduction of this feature with increasing temperature is due to the disappearance of heptagons. The arrow in panel (b) indicates the same feature in our data, and we similarly observe this feature disappearing with temperature. We inspected our
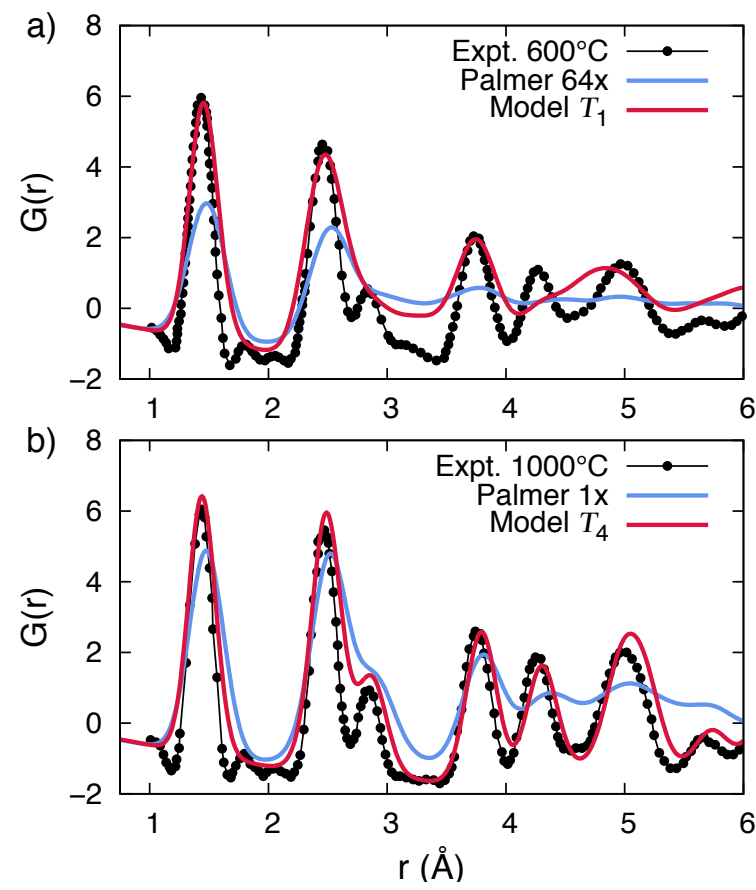

Figure 5: Comparison between experimental and simulated pair distribution functions (PDFs). The simulated PDFs have been convolved with a Gaussian of width $0.11 \AA$. Experimental data from Forse et al. [57] is shown as solid black circles. The simulation data from Palmer et al. [32] and this work are shown as blue and red lines, respectively. As described in the text, panels a) and b) show a comparison for low and high synthesis temperature, respectively. 
structures, and confirmed that distances around $3.2 \AA$ are indeed associated with third-neighbour distances in heptagons. This observation is supported by our ring statistics in Figure 3 which shows heptagons progressively disappearing as temperature increases.

Figure 5 compares our data with experimental data from Forse et al. [57] and two models from Palmer et al. [32] As noted in the methodology, all computational data is convolved with a Gaussian to facilitate comparison with the experimental data. The convolution process is necessary due to the finite maximum scattering vector $Q_{\max }$ in the experiment and transforms the raw data from Figure 4 into the data shown as red lines in Figure 5. The same process has been applied to the raw data from Palmer et al. Panel (a) compares our model $T_{1}$ with experimental data at a low synthesis temperature of $600{ }^{\circ} \mathrm{C}$. Palmer et al. identified their most rapidly quenched structure $(64 \times)$ to correspond to this temperature. While not perfect, our model is a major improvement over the Palmer model, reproducing the height of the first two peaks and much of the structure of the third and subsequent neighbours. In contrast, the model of Palmer et al. is much more disordered and there is minimal structure beyond the second neighbour. A similar comparison is made in panel (b) for high temperature. The experimental data corresponds to the highest temperature available and shows long-range order extending out to approximately $15 \AA$. We compare it to model $T_{4}$ which exhibits order to a similar extent and to the slowest quench rate $(1 \times)$ by Palmer et al. The agreement between our model and the experimental data is excellent, reproducing the peak height and position of the first seven neighbours. No previous mimetic model has produced this level of agreement. The model of Palmer et al. performs better than at low temperature, reproducing more peaks, but there is little structure beyond the fourth peak.

One of the critical characteristics of CDCs is their porosity, which is defined as the empty volume contained within the structure. Experimentally, it has been observed that the total porosity is similar for all temperatures while the distribution of pores is temperature dependent. [10] Figure 6 shows the full TiC-CDC structure annealed at $T_{4}$, with the pore sizes calculated using the largest fitting sphere [54] and shown as grey spheres. Applying this method for our structures yields a porosity between 58.4 and $59.4 \%$, in good agreement with the estimated

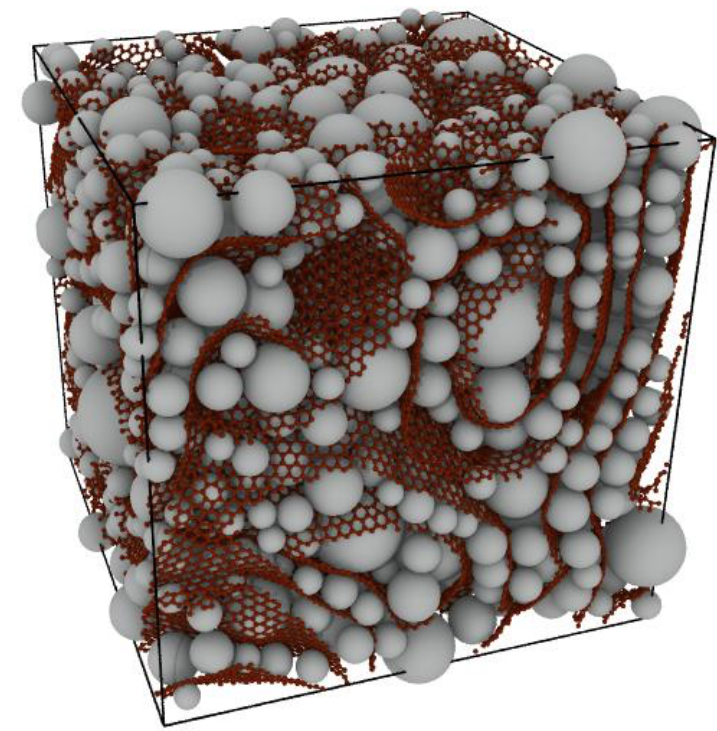

Figure 6: $3 \mathrm{D}$ image of the TiC-CDC simulated at $T_{4}=$ $4000 \mathrm{~K}$. Red balls and sticks represent the carbon atoms and their bonds, respectively. Grey spheres indicate pores calculated with the sphere-pore model. [54] The simulation box is depicted with black lines to guide the eye.

value of $57 \%$. $[2,6]$ In $\mathrm{TiC}-\mathrm{CDCs}$, mesopores are created by voids between stacked graphitic ribbons [8]. As we can observe in our structure, the largest pores are located between groups of stacked graphene layers. However, the lack of attractive forces between layers in the EDIP potential means the inter-layer spacing is not fixed at around $3.35 \AA$ and instead the spacing is driven by the density and the connectivity of the microstructure. The lack of long-range attraction allows the opening up of spaces between the layers which can be accessed by the gas molecules. An example of this effect is seen at the bottom-right of Figure 6 where small grey spheres of diameter $\sim 5 \AA$ appear between stacked layers. This behaviour is clearly not ideal and suggests that useful future improvements on our model can be achieved by including attractive Van der Waals forces in the EDIP functional form.

The porosity is often quantified experimentally by gas adsorption isotherms and so it is useful to calculate the isotherm of our simulated structures. Figure 7 compares our Ar adsorption isotherms calculated using GCMC with experimental data from Dash et al. [6] and GCMC isotherms from Palmer et al. [32] All of the GCMC isotherms show a marked type-I behavior typical of microporous carbons [60] in which the adsorption saturates at low relative 
pressure. At low temperature, shown in panel (a), we observe excellent agreement between our model $T_{1}$ and experiment, while the model of Palmer et al. underestimates the adsorption capacity by $50 \%$. At high temperature, shown in panel (b), neither of the models agree with the experiment and the simulated isotherms saturate while the experimental isotherm continues to increase up until atmospheric pressure. The same discrepancy has been seen in HRMC models by Farmahini et al. [? ] where the simulated isotherms also plateau and underestimate the total adsorption capacity by around $30 \%$.

Figure 8 shows a semi-logarithmic plot of the low pressure range and includes experimental data as available. Our model $T_{1}$ corresponding to the low synthesis temperature [panel (a)] is in excellent agreement with the experiment, confirming that the good agreement seen in Figure 7 extends over the entire pressure range. Pore filling starts at very low
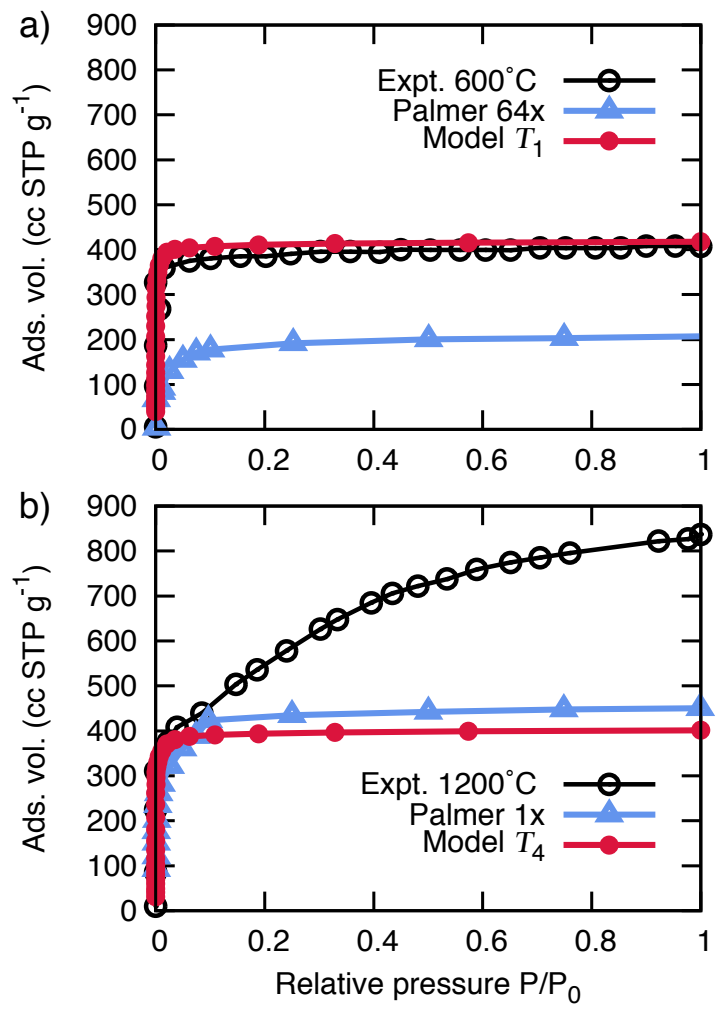

Figure 7: Comparison between experimental and simulated Ar adsorption isotherms at $77 \mathrm{~K}$. Experimental data from Dash et al. [6] is shown as black circles. Simulation data from Palmer et al. [32] and this work are shown as blue and red lines, respectively. As described in the text, panels a) and b) show a comparison for low and high synthesis temperature, respectively. pressure $\left(P / P_{0} \sim 10^{-7}\right)$, indicating the presence of large number of narrow micropores as intensive adsorption spaces. In contrast, the corresponding model of Palmer et al. exhibits almost no adsorption. In panel (b) our model $T_{2}$ slightly overpredicts the adsorption but still maintains a good trend in agreement with the experiment. The overprediction increases with temperature as seen in panels (c) and (d). This indicates an excess of micropores in the structures and in the case of model $T_{4}$ can be attributed to the wide spacing between graphitic layers as discussed above.

Determining the pore size distribution (PSD) is an important, yet challenging, task. Experimentally, the PSD is not measured directly, but instead is inferred from adsorption isotherms using non-local density functional theory (NLDFT) [14] or quenched solid density functional theory (QSDFT). [61] Although QSDFT is more accurate, here we present NLDFT calculations in order to compare with experiment. Another route to calculating the PSD is directly from the atomic coordinates themselves, using the method of non-overlapping spheres as seen earlier in Figure 6. With the isotherm/DFT methods the result is dependent on the choice of the adsorbate gas species, while the geometric PSD has no such dependence.

Figure 9 compares NLDFT data derived from experimental isotherms (black lines) [6], NLDFT data derived from our simulated isotherms (red lines) as well as our geometrically calculated PSD (green lines). For model $T_{1}$, seen in panel (a), there is reasonable agreement between the experimental and computational NLDFT data. The geometric PSD does not show the sharp peaks or minima that appear in the NLDFT data, and the pores as defined geometrically extend to slightly larger sizes. Note that the sharp minima at $\sim 1 \mathrm{~nm}$ in the NLDFT data is a well-known artifact of the NLDFT method [61]. For model $T_{2}$, seen in panel (b), the experimental and computational NLDFT data are in excellent agreement up to $1.1 \mathrm{~nm}$, but for the rest of the range, only the geometric PSD is in agreement. A similar situation applies for model $T_{3}$. For model $T_{4}$, seen in panel (d), the situation changes and the computational NLDFT data yields an excess of micropores in the range of $0.4-0.7 \mathrm{~nm}$. This is due to pore filling between graphitic layers as discussed in reference to Figure $8(\mathrm{~d})$. Over the range $0.7-1.2 \mathrm{~nm}$ the agreement between all three methods is good, while from $1.2 \mathrm{~nm}$ onwards only the geometric PSD shows the existence of large pores as observed in the 


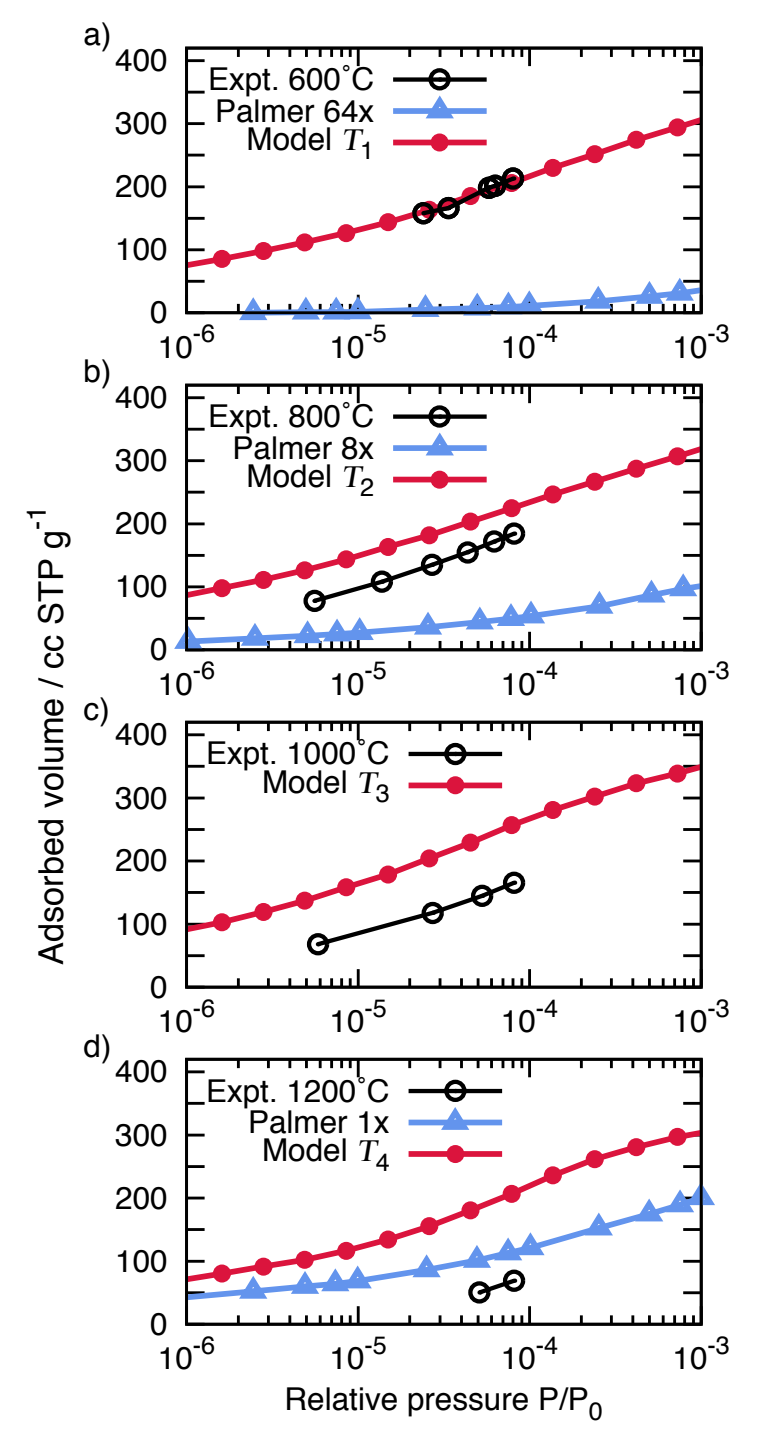

Figure 8: Low pressure range of the experimental and simulated Ar adsorption isotherms at $77 \mathrm{~K}$ at different synthesis temperatures. Experimental data, when available, from Dash et al. [6] is shown as black circles. Simulation data from Palmer et al. [32] and this work are shown as blue and red lines, respectively.

experimental DFT.

It is worth noting that the geometric PSD shows an increase in maximum pore size with temperature as seen in the experimental NLDFT data. At low temperature the maximum pore size computed geometrically is $1.5 \mathrm{~nm}$, increasing to $2 \mathrm{~nm}$ at intermediate temperatures, and reaching $2.85 \mathrm{~nm}$ at the highest temperature. This is much larger than the maximum pore size of $1.7 \mathrm{~nm}$ reported by Palmer et al. This likely reflects their small system size of around 4000 atoms, which precludes the possibility of large pores. A significant size-effect was seen in the study of ZrC-CDCs by Lopez and coworkers [62] where a 6912-atom system had a maximum pore size of $2.1 \mathrm{~nm}$, while for a 62,500 -atom system pores up to $3.0 \mathrm{~nm}$ were observed. Ranganathan et al. [39] also found a system-size effect in their ReaxFF simulations. This highlights the

a)

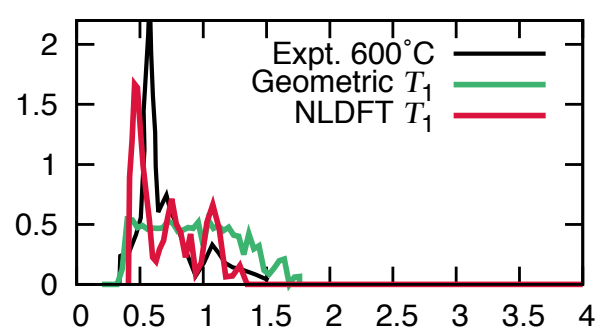

b)

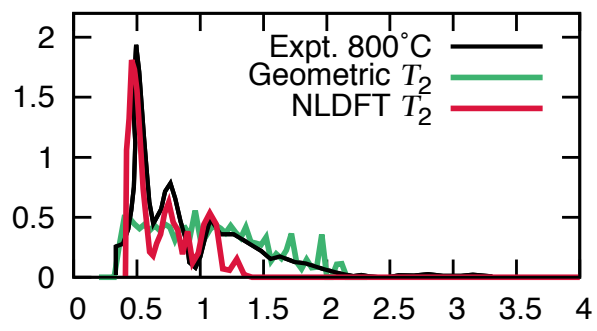

c)

ญ

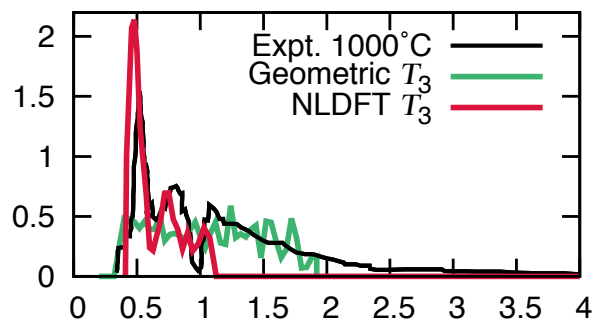

d)

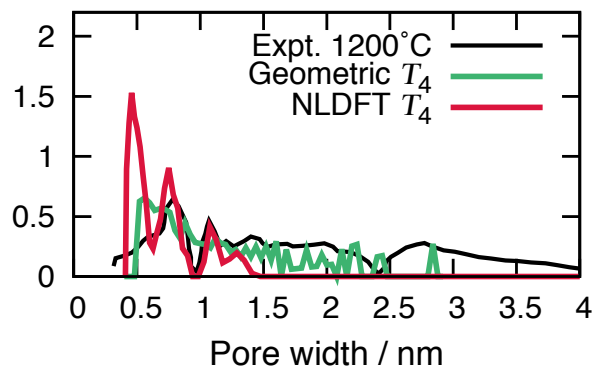

Figure 9: Pore size distributions (PSD) of TiC-CDCs generated at different temperatures. Black lines show the PSDs calculated with NLDFT from the experimental Ar adsorption isotherms from Ref. [6]. Red lines show the PSDs calculated with NLDFT from the computed Ar adsorption isotherms. Green lines show the PSDs calculated directly from the atomistic coordinates using a geometric method. 
importance of using very large system sizes, containing many hundreds of thousands of atoms, to describe the mesoporosity.

\section{Conclusion}

We have developed a mimetic model of CDC synthesis with an improved ability to predict experimental properties. Achievements of our model include: (i) the ability to successfully predict graphitization with increasing synthesis temperature, (ii) coordination numbers in agreement with EELS data, (iii) pair distribution functions which reproduce long-range order and trends seen in experimental scattering data, (iv) the observation of heptagons in the structure which disappear with increasing temperature, as suggested by Forse et al., and $(\mathrm{v})$ the ability to predict adsorption properties in the micropore range. While our model is not perfect, it is a significant improvement over existing mimetic models which have not predicted graphitization and compare poorly to experiment for many of the quantities listed above.

The strength of our simulation approach lies in the use of the Environment Dependent Interaction Potential (EDIP) [41], a large system size and annealing for long times. The high transferability of EDIP results in a good prediction of most properties, with the exception of the interlayer stacking distance, which results in an excess of micropores at high temperatures. Our large system size allows the formation of micropores and small mesopores, but to achieve large mesopores would require circa 10-20 times more atoms and an interatomic potential with long-range attraction. The use of longtime (2 ns) annealing is a central part of our model that enables evolution of the structure towards a graphitic structure. This evolution takes time and is activated by temperature, and therefore cannot be seen in quenching methods. Furthermore, the constant temperature of the annealing process allows an intuitive link between simulation and experimental temperatures via an Arrhenius correlation. This correlation is beneficial as it enables MD simulations operating on the nanosecond scale to be related to experiments running for hours. Additionally, the activation barriers obtained through the Arrhenius equation add physical meaning to the mechanisms; the barriers are the same order of magnitude as atomistic rearrangements of graphite defects. Since our approach is both mimetic and predictive, it can in principle be applied to any CDC precursor which graphitizes in the absence of a catalyst.

\section{Acknowledgments}

This work was supported by Australian Research Council (DP150103487). NAM and ISM acknowledge fellowships FT120100924 and FT140100191, respectively. Computational resources are provided by the Pawsey Supercomputing Centre with funding from the Australian Government and the Government of Western Australia. MJL acknowledges MICINN of Spain (Grant MAT2014-54378-R) and Junta de Castilla y Leon (Grant VA050U14). FVB and KK acknowledge project JSTCREST Creation of Innovative Functional Materials with Advanced Properties by Hyper-nano-space Design.

\section{References}

[1] J. Chmiola, C. Largeot, P.-L. Taberna, P. Simon, Y. Gogotsi, Monolithic carbide-derived carbon films for micro-supercapacitors, Science 328 (2010) 480-483. doi:10.1126/science.1184126.

[2] V. Presser, M. Heon, Y. Gogotsi, Carbide-Derived Carbons - From Porous Networks to Nanotubes and Graphene, Adv. Funct. Mater. 21 (2011) 810-833. doi:10.1002/adfm.201002094.

[3] C. Merlet, B. Rotenberg, P. A. Madden, P.-L. Taberna, P. Simon, Y. Gogotsi, M. Salanne, On the molecular origin of supercapacitance in nanoporous carbon electrodes, Nat. Mater. 11 (4) (2012) 306-310. doi:10.1038/NMAT3260.

[4] G. N. Yushin, E. N. Hoffman, A. Nikitin, H. Ye, M. W. Barsoum, Y. Gogotsi, Synthesis of nanoporous carbide-derived carbon by chlorination of titanium silicon carbide, Carbon 43 (2005) 2075-2082. doi:10.1016/j.carbon.2005.03.014.

[5] R. K. Dash, G. Yushin, Y. Gogotsi, Synthesis, structure and porosity analysis of microporous and mesoporous carbon derived from zirconium carbide, Microporous Mesoporous Mat. 86 (2005) 50-57. doi:10.1016/j.micromeso.2005.05.047.

[6] R. Dash, J. Chmiola, G. Yushin, Y. Gogotsi, G. Laudisio, J. Singer, J. Fischer, S. Kucheyev, Titanium carbide derived nanoporous carbon for energyrelated applications, Carbon 44 (2006) 2489-2497. doi:10.1016/j.carbon.2006.04.035.

[7] G. Yushin, R. Dash, J. Jagiello, J. E. Fischer, Y. Gogotsi, Carbide-derived carbons: Effect of pore size on hydrogen uptake and heat of adsorption, Adv. Funct. Mater. 16 (2006) 2288-2293. doi:10.1002/adfm.200500830.

[8] J. Gläsel, J. Diao, Z. Feng, M. Hilgart, T. Wolker, D. S. $\mathrm{Su}$, B. J. M. Etzold, Mesoporous and graphitic carbidederived carbons as selective and stable catalysts for the dehydrogenation reaction, Chem. Mater. 27 (2015) 5719-5725. doi:10.1021/acs.chemmater.5b02262.

[9] J. Chmiola, G. Yushin, R. Dash, Y. Gogotsi, Effect of pore size and surface area of carbide derived carbons on specific capacitance, J. Power Sources 158 (2006) 765772. doi:10.1016/j.jpowsour.2005.09.008.

[10] A. M. Kern, B. Zierath, J. Haertlé, T. Fey, B. J. M. 
Etzold, Thermal and Electrical Conductivity of Amorphous and Graphitized Carbide-Derived Carbon Monoliths, Chem. Eng. Technol. 39 (2016) 1121-1129. doi:10.1002/ceat.201600011.

[11] T. J. Bandosz, M. J. Biggs, K. E. Gubbins, Y. Hattori, T. Iiyama, K. Kaneko, et al., Chemistry and physics of carbon, Vol. 28, Marcel Dekker, New York, 2003.

[12] P. J. F. Harris, Structure of non-graphitising carbons, Int. Mater. Rev. 42 (1997) 206-218. doi:10.1179/imr.1997.42.5.206.

[13] M. Biggs, P. Agarwal, Mass diffusion of atomic fluids in random micropore spaces using equilibrium molecular dynamics, Phys. Rev. A 46 (1992) 3312-3318.

[14] C. Lastoskie, K. E. Gubbins, N. Quirke, Pore size heterogeneity and the carbon slit pore: a density functional theory model, Langmuir 9 (1993) 2693-2702. doi:10.1021/la00034a032.

[15] T. X. Nguyen, S. K. Bhatia, Characterization of pore wall heterogeneity in nanoporous carbons using adsorption: the slit pore model revisited, J. Phys. Chem. B 108 (2004) 14032-14042. doi:10.1021/jp049048f.

[16] A. Wongkoblap, D. D. Do, Adsorption of water in finite length carbon slit pore: Comparison between computer simulation and experiment, J. Phys. Chem. B 111 (2007) 13949-13956. doi:10.1021/jp0747297.

[17] J. Jagiello, J. P. Olivier, Carbon slit pore model incorporating surface energetical heterogeneity and geometrical corrugation, Adsorption 19 (2013) 777-783. doi:10.1007/s10450-013-9517-4.

[18] J. Jagiello, J. P. Olivier, 2D-NLDFT adsorption models for carbon slit-shaped pores with surface energetical heterogeneity and geometrical corrugation, Carbon 55 (2013) 70-80. doi:10.1016/j.carbon.2012.12.011.

[19] D. D. Do, H. D. Do, Modeling of adsorption on nongraphitized carbon surface: GCMC simulation studies and comparison with experimental Data, J. Phys. Chem B 110 (2006) 17531-17538. doi:10.1021/jp062386r.

[20] M. J. Biggs, A. Buts, D. Williamson, Absolute assessment of adsorption-based porous solid characterization methods: comparison methods, Langmuir 20 (2004) 7123-7138. doi:10.1021/la036435s.

[21] Q. Cai, A. Buts, M. J. Biggs, N. A. Seaton, Evaluation of methods for determining the pore size distribution and pore-network connectivity of porous carbons, Langmuir 23 (2007) 8430-8440. doi:10.1021/la7007057.

[22] P. J. F. Harris, S. C. Tsang, High-resolution electron microscopy studies of non-graphitizing carbons, Phil. Mag. A 76 (1997) 667-677. doi:10.1080/01418619708214028.

[23] M. Acharya, M. S. Strano, J. P. Mathews, S. J. L. Billinge, V. Petkov, S. Subramoney, H. C. Foley, Simulation of nanoporous carbons: A chemically constrained structure, Philos. Mag. B 79 (1999) 1499-1518. doi:10.1080/13642819908218318.

[24] S. K. Bhatia, Characterizing Structural Complexity in Disordered Carbons: From the Slit Pore to Atomistic Models, Langmuir 33 (2017) 831-847. doi:10.1021/acs.langmuir.6b03459.

[25] G. Opletal, T. Petersen, B. O'Malley, I. Snook, Hybrid approach for generating realistic amorphous carbon structure using metropolis and reverse Monte Carlo, Mol. Sim. 28 (2002) 927-938. doi:10.1080/089270204000002584.

[26] S. K. Jain, R. J. M. Pellenq, J. P. Pikunic, K. E. Gubbins, Molecular modeling of porous carbons Using the hybrid reverse Monte Carlo method, Langmuir 22 (2006) 9942-9948. doi:10.1021/la053402z.

[27] G. Opletal, T. C. Petersen, D. G. McCulloch, I. K. Snook, I. Yarovsky, The structure of disordered carbon solids studied using a hybrid reverse Monte Carlo algorithm, J. Phys.: Condens. Matter 17 (2005) 2605-2616. doi:10.1088/0953-8984/17/17/008.

[28] T. X. Nguyen, S. K. Bhatia, S. K. Jain, K. E. Gubbins, Structure of saccharose-based carbon and transport of confined fluids: hybrid reverse Monte Carlo reconstruction and simulation studies, Mol. Sim. 32 (2007) 567577. doi:10.1080/08927020600675699.

[29] P. Zetterström, S. Urbonaite, F. Lindberg, R. G. Delaplane, J. Leis, G. Svensson, Reverse Monte Carlo studies of nanoporous carbon from TiC, J. Phys. Condens. Matter 17 (2005) 3509-3524. doi:10.1088/0953$8984 / 17 / 23 / 004$.

[30] P. Kowalczyk, A. P. Terzyk, P. A. Gauden, S. Furmaniak, M. Winiewski, A. Burian, L. Hawelek, K. Kaneko, A. V. Neimark, Carbon molecular sieves: Reconstruction of atomistic structural models with experimental constraints, J. Phys. Chem. C 118 (24) (2014) 1299613007. doi:10.1021/jp503628m.

[31] A. H. Farmahini, S. K. Bhatia, Hybrid Reverse Monte Carlo simulation of amorphous carbon: Distinguishing between competing structures obtained using different modeling protocols, Carbon 83 (2015) 53-70. doi:10.1016/j.carbon.2014.11.013.

[32] J. C. Palmer, A. Llobet, S. H. Yeon, J. E. Fischer, Y. Shi, Y. Gogotsi, K. E. Gubbins, Modeling the structural evolution of carbide-derived carbons using quenched molecular dynamics, Carbon 48 (2010) 11161123. doi:10.1016/j.carbon.2009.11.033.

[33] Y. Shi, A mimetic porous carbon model by quench molecular dynamics simulation, J. Chem. Phys. 128 (2008) 234707-11. doi:10.1063/1.2943645.

[34] L. J. Peng, J. R. Morris, Structure and hydrogen adsorption properties of low density nanoporous carbons from simulations, Carbon 50 (2012) 1394-1406. doi:10.1016/j.carbon.2011.11.012.

[35] A. Kumar, R. F. Lobo, N. J. Wagner, Porous amorphous carbon models from periodic Gaussian chains of amorphous polymers, Carbon 43 (2005) 3099-3111. doi:10.1016/j.carbon.2005.06.030.

[36] S. Furmaniak, New virtual porous carbons based on carbon EDIP potential and Monte Carlo simulations, Comput. Methods Sci. Technol. 19 (2013) 47-57. doi:10.12921/cmst.2013.19.01.47-57.

[37] M. J. López, I. Cabria, J. A. Alonso, Simulated porosity and electronic structure of nanoporous carbons, J. Chem. Phys. 135 (2011) 104706-10. doi:10.1063/1.3633690.

[38] K. Nordlund, J. Keinonen, T. Mattila, Formation of Ion Irradiation Induced Small-Scale Defects on Graphite Surfaces, Phys. Rev. Lett. 77 (1996) 699-702. doi:10.1103/PhysRevLett.77.699.

[39] R. Ranganathan, S. Rokkam, T. Desai, P. Keblinski, Generation of amorphous carbon models using liquid quench method: A reactive molecular dynamics study, Carbon 113 (2017) 87-99. doi:10.1016/j.carbon.2016.11.024.

[40] A. C. T. van Duin, S. Dasgupta, F. Lorant, W. A. Goddard, ReaxFF: A Reactive Force Field for Hydrocarbons, J. Phys. Chem. A 105 (2001) 9396-9409. doi:10.1021/jp004368u. 
[41] N. A. Marks, Generalizing the environment-dependent interaction potential for carbon, Phys. Rev. B 63 (2000) 035401. doi:10.1103/PhysRevB.63.035401.

[42] C. De Tomas, I. Suarez-Martinez, N. A. Marks, Graphitization of amorphous carbons: A comparative study of interatomic potentials, Carbon 109 (2016) 681-693. doi:10.1016/j.carbon.2016.08.024.

[43] I. Suarez-Martinez, P. J. Higginbottom, N. A. Marks, Molecular dynamics simulations of the transformation of carbon peapods into double-walled carbon nanotubes, Carbon 48 (2010) 3592-3598. doi:10.1016/j.carbon.2010.06.004.

[44] I. Suarez-Martinez, N. A. Marks, Amorphous carbon nanorods as a precursor for carbon nanotubes, Carbon 50 (2012) 5441-5449. doi:10.1016/j.carbon.2012.07.030.

[45] R. C. Powles, N. A. Marks, D. W. M. Lau, Selfassembly of sp2-bonded carbon nanostructures from amorphous precursors, Phys. Rev. B 79 (2009) 07543011. doi:10.1103/PhysRevB.79.075430.

[46] D. W. M. Lau, A. Moafi, M. B. Taylor, J. G. Partridge, D. G. McCulloch, R. C. Powles, D. R. McKenzie, The structural phases of non-crystalline carbon prepared by physical vapour deposition, Carbon 47 (2009) 32633270. doi:10.1016/j.carbon.2009.07.044.

[47] S. Furmaniak, S. Koter, A. P. Terzyk, P. A. Gauden, P. Kowalczyk, G. Rychlicki, New insights into the ideal adsorbed solution theory, Phys. Chem. Chem. Phys. 17 (2015) 7232-7247. doi:10.1039/c4cp05498a.

[48] S. Plimpton, Fast parallel algorithms for short-range molecular dynamics, J. Chem. Phys. 117 (1995) 1-19. doi:10.1006/jcph.1995.1039.

[49] G. Bussi, D. Donadio, M. Parrinello, Canonical sampling through velocity rescaling, J. Chem. Phys. 126 (2007) 014101. doi:10.1063/1.2408420.

[50] D. S. Franzblau, Computation of ring statistics for network models of solids, Phys. Rev. B 44 (10) (1991) 4925-4930. doi:10.1103/PhysRevB.44.4925.

[51] N. A. Marks, N. C. Cooper, D. R. McKenzie, D. G. McCulloch, P. Bath, S. P. Russo, Comparison of densityfunctional, tight-binding, and empirical methods for the simulation of amorphous carbon, Phys. Rev. B 65 (7) (2002) 075411. doi:10.1103/PhysRevB.65.075411.

[52] A. Stukowski, Visualization and analysis of atomistic simulation data with OVITO: the Open Visualization Tool, Modell. Simul. Mater. Sci. Eng. 18 (2010) 015012.

[53] B. Coasne, S. K. Jain, L. Naamar, K. E. Gubbins, Freezing of argon in ordered and disordered porous carbon, Phys. Rev. B 76 (2007) 085416. doi:10.1103/PhysRevB.76.085416.

[54] S. Bhattacharya, K. E. Gubbins, Fast method for computing pore size distributions of model materials, Langmuir 22 (2006) 7726-7731. doi:10.1021/la052651k

[55] Quantachrome, http://www.quantachrome.com/technical/dft.html.

[56] S. Ishikawa, T. Saito, K. Kuwahara, Carbon materials with nano-sized pores derived from carbides, SEI Technical Review (2016) 153.

[57] A. C. Forse, C. Merlet, P. K. Allan, E. K. Humphreys, J. M. Griffin, M. Aslan, M. Zeiger, V. Presser, Y. Gogotsi, C. P. Grey, New insights into the structure of nanoporous carbons from NMR, Raman, and pair distribution function analysis, Chem. Mater. 27 (2015) 6848-6857. doi:10.1021/acs.chemmater.5b03216.

[58] N. A. Marks, M. F. Cover, C. Kocer, Simulating temperature effects in the growth of tetrahedral amorphous carbon: The importance of infrequent events, App.
Phys. Lett. 89 (2006) 131924-3. doi:10.1063/1.2358116. [59] C. D. Latham, A. J. McKenna, T. P. Trevethan, M. I. Heggie, M. J. Rayson, P. R. Briddon, On the validity of empirical potentials for simulating radiation damage in graphite: a benchmark, J. Phys.: Condens. Matter 27 (2015) 316301. doi:10.1088/0953-8984/27/31/316301.

[60] S. J. Gregg, K. S. W. Sing, Adsorption, surface area, and porosity, Academic Press, London, 1982.

[61] A. V. Neimark, Y. Lin, P. I. Ravikovitch, M. Thommes, Quenched solid density functional theory and pore size analysis of micro-mesoporous carbons, Carbon 47 (2009) 1617-1628. doi:10.1016/j.carbon.2009.01.050.

[62] J. A. Alonso, I. Cabria, M. J. López, Simulation of hydrogen storage in porous carbons, J. Mater. Research 28 (2012) 589-604. doi:10.1557/jmr.2012.370. 